\begin{abstract}
TONGTONG, CHEN. A MIMO Receiver SOC for CDMA Applications. (Under the direction of Professor XUN LIU).

Multiple Input Multiple Output(MIMO) technique promises substantial increase of wireless channel capacity by using antenna arrays at both transmitters and receivers. It is one of the key technology to be used in the third generation wireless communication applications and is a current theme of international wireless research. Hardware implementation of MIMO receiver in today's wireless applications has stringent requirements such as high throughput, low power and high performance. This brings the difficulties to carry out the desired ASIC chip which is feasible to current silicon process.

In this thesis, we introduce a new System-on-a-Chip(SoC) design for the $3 \mathrm{G}$ Code Division Multiple Access(3G-CDMA) MIMO receiver. The SoC chip consists of a space-time equalizer, a MIMO detector and a turbo decoder onto a single chip, which can be configured to handle different modulation schemes including QPSK and 16QAM according to the signal-to-noise ratio(SNR). At low SNR, QPSK modulation scheme can provide lower bit error rate(BER), while at high SNR, 16QAM scheme can have a larger throughput. Sphere decoding algorithm is used for MIMO detection to achieve near maximum likelihood (ML) performance with relatively lower complexity for practical silicon implementation. To improve the system performance further, we implement a turbo decode, which decode the transmitted information bits using the soft decision result from the sphere decoder. Our design can achieve much lower BER than other current MIMO ASICs in the low SNR range. The paper also analyze the trade-off between the hardware complexity and the BER performance of the MIMO receiver using MATLAB fixed-point simulation and hardware synthesis.
\end{abstract}




\title{
A MIMO Receiver SOC for CDMA Applications
}

\author{
by \\ Tongtong Chen \\ A thesis submitted to the Graduate Faculty of \\ North Carolina State University \\ in partial fulfillment of the \\ requirements for the Degree of \\ Master of Science
}

Electrical Engineering

Raleigh, North Carolina

2007

Approved By:

Dr. W. Rhett Davis

Dr. Paul D. Franzon

Dr. Xun Liu

Chair of Advisory Committee 


\section{Dedication}

To my wife

Shan Huang

and my parents

\section{Jianzhong Chen and Ruoju Huang}




\section{Biography}

Tongtong Chen was born in Zhanjiang, P.R.China in 1981. He received his B.S. degree in Microelectronics from Peking University, Beijing, China in 2002. He joined Arcadia Design System Inc. in Shanghai after graduation from college and worked for two and half years as physical designer. In Fall 2005, he began his graduate studies in the Electrical and Computer Engineering Department at North Carolina State University, Raleigh, NC. He has been working with Dr. Xun Liu in the area of hardware design for wireless communication system. 


\section{Acknowledgements}

I would like to sincerely thank my adviser, Dr. Xun Liu, for his guidance, support and encouragement throughout my graduate studies. He gave me an opportunity to work for him and inspirited me with creative ideas for my research. His patience with me make this thesis possible.

I also thank my committee members: Dr. Paul D. Franzon and Dr. W. Rhett Davis for their support and guidance on my course works and research studies.

I greatly appreciate the help given to me by my group mates, Zhentao Yu, Yuantao Peng and Tamin Kim, for sharing their knowledge and experience with me and helping me with a lot of design issues on my research topic.

Finally, a special thanks is given to my beloved wife, Shan Huang, for always being with me and supporting me during these past three years in the United States of America. I also thank my parents and my two sisters for their love and support. 


\section{Contents}

List of Figures $\quad$ vii

List of Tables

1 Introduction $\quad 1$

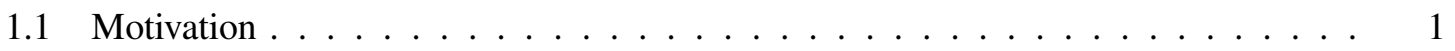

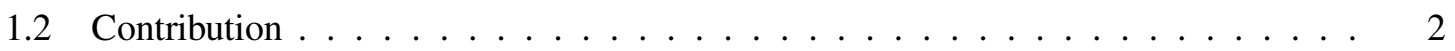

1.3 Thesis Organization . . . . . . . . . . . . . . . . . 3

2 Background and Related Work 4

2.1 Background . . . . . . . . . . . . . . . . . . . . 4

2.1.1 MIMO CDMA Communication Architecture . . . . . . . . . . . . 4

2.1.2 MIMO Channel Model . . . . . . . . . . . . . . . . . . . . 5

2.1 .3 Turbo Decoding . . . . . . . . . . . . . . . 6

2.2 Previous MIMO Receiver Design . . . . . . . . . . . . . . . . 8

3 Overview of MIMO Receiver Design 9

4 RTL Design of Equalization and Flat Channel Estimation Module 10

4.1 Equalization Module . . . . . . . . . . . . . . . . . . . . 10

4.1 .1 FIR Filter . . . . . . . . . . . . . . . . . . . 11

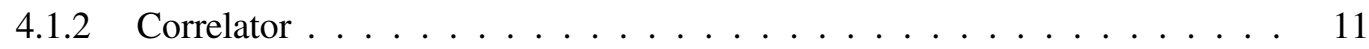

4.1 .3 C-SS Pilot Generator . . . . . . . . . . . . . . . . . . 11

4.1.4 NLMS Error Calculation and FIR Tap Updating . . . . . . . . . . . . . . . 12

4.2 Flat Channel Estimation Module . . . . . . . . . . . . . . . . . . . . . . . . . . . . . . . .

4.3 Verification Procedure . . . . . . . . . . . . . . . . 13

5 RTL Design of Sphere Decoder Module 15

5.1 Overview of Sphere Decoder Module Design _ . . . . . . . . . . . . . . . 15

5.2 Sphere Decoding Searching Procedure . . . . . . . . . . . . . . . 16

5.3 Block Diagram of the Sphere Decoder . . . . . . . . . . . . . . . . . . 16

5.3.1 QR Decomposition and Unconstrained Symbol Computation . . . . . . . . 17

5.3.2 Matrix computation . . . . . . . . . . . . . . . . 18 
5.3 .3 Matrix Enumeration . . . . . . . . . . . . . . . . . . 18

5.3 .4 Book Keeping . . . . . . . . . . . . . . . . . . . . . . . . 18

5.4 Verification Procedure $\ldots \ldots \ldots \ldots \ldots \ldots$

6 RTL Design of Turbo Decoder 21

6.1 Overview of Turbo Decoder Design . . . . . . . . . . . . . . . . . 21

6.2 Detail Data Flow in the Turbo Decoder . . . . . . . . . . . . . . . . . . . . . 22

6.3 The Operation of the Sliding Windows . . . . . . . . . . . . . . . 23

6.4 Detail Sub-module Design . . . . . . . . . . . . . . . . . . 23

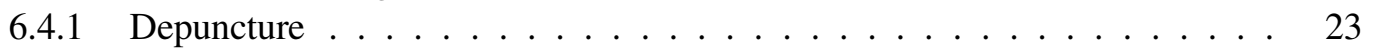

6.4 .2 Transition Metric Unit . . . . . . . . . . . . . . . . . . . . . . 24

6.4.3 Forward and Backward Metric Unit . . . . . . . . . . . . . . . . 24

6.4 .4 LLR Unit . . . . . . . . . . . . . . . . . . . . . . . 25

6.4 .5 Interleaving ROM and RAM . . . . . . . . . . . . . . 25

6.4 .6 Control Logic . . . . . . . . . . . . . . . . . . . . . . 26

6.5 Verification Procedure $\ldots \ldots \ldots \ldots \ldots \ldots \ldots$

7 Optimization Scheme $\quad 30$

7.1 Theoretical Hardware Complexity Analysis . . . . . . . . . . . . . . . . 30

7.2 Critical Signals . . . . . . . . . . . . . . . . . . . . . 31

$7.3 \quad L_{c}$ Value in Turbo Decoder $\ldots \ldots \ldots \ldots \ldots \ldots \ldots$

8 Chip Design Result $\quad 35$

8.1 Design Flow . . . . . . . . . . . . . . . . . . . 35

8.2 Chip Performance and Layout Summary . . . . . . . . . . . . . . . . 35

9 Conclusions and Future Work $\quad 38$

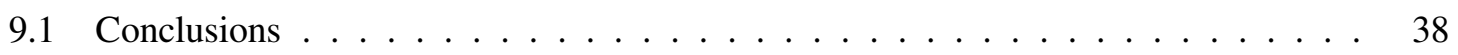

9.2 Future Work . . . . . . . . . . . . . . . . . . . 38

$\begin{array}{ll}\text { Bibliography } & 39\end{array}$ 


\section{List of Figures}

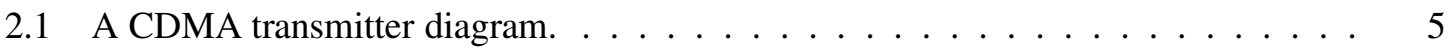

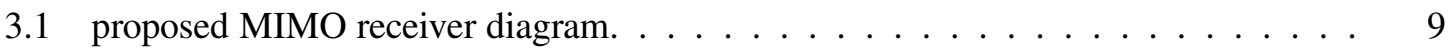

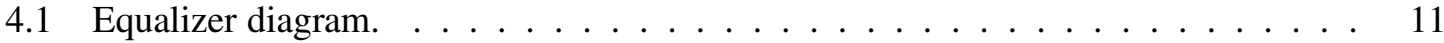

4.2 FIR block diagram. . . . . . . . . . . . . . . . . . . . . . . 12

4.3 Correlator block diagram. . . . . . . . . . . . . . . . . . . . . 13

4.4 LFSR block diagram. . . . . . . . . . . . . . . . . . . . . . . . . . 13

4.5 Weight update block diagram. . . . . . . . . . . . . . . . . 14

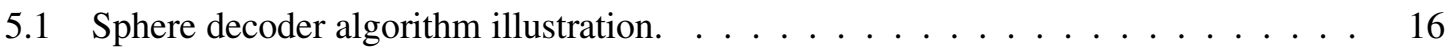

5.2 Sphere decoder diagram. . . . . . . . . . . . . . . . . . . . . 17

5.3 Matrix computation block diagram. . . . . . . . . . . . . . 18

5.4 Matrix enumeration block diagram. . . . . . . . . . . . . . . . . . . . 19

5.5 Book keeping recording diagram. . . . . . . . . . . . . . . . . . . . . 19

6.1 Turbo decoder. . . . . . . . . . . . . . . . . . . . 22

6.2 forward and backward metric block diagram. . . . . . . . . . . . . . . 25

6.3 LLR calculation block diagram. . . . . . . . . . . . . . . . . 28

6.4 Control logic FSM . . . . . . . . . . . . . . . . . . . . . . . . . . . . 29

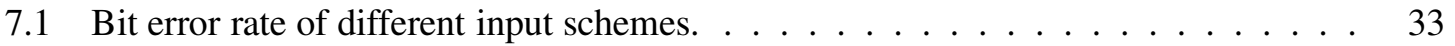

7.2 Bit error rate of different LLR schemes. . . . . . . . . . . . . . . . . . . . . 34

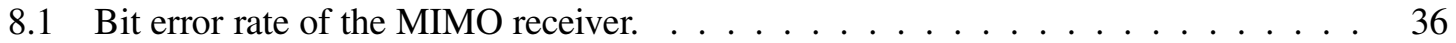

8.2 Full chip layout. . . . . . . . . . . . . . . . . . . . . . . 37 


\section{List of Tables}

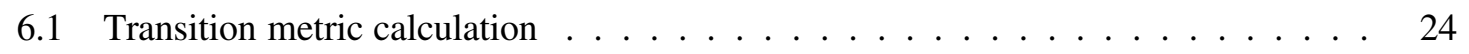

7.1 Sphere Decoder Quantization Summary. . . . . . . . . . . . . . . . . . . . 31

7.2 Input Signal Quantization Summary. . . . . . . . . . . . . . . . . . . 32

7.3 LLR Quantization Summary. . . . . . . . . . . . . . . . . . . . 32 


\section{Chapter 1}

\section{Introduction}

\subsection{Motivation}

MIMO technology is a scheme that promises substantial increase of wireless channel capacity by deploying antenna arrays at both transmitters and receivers. The signals are transmitted in multiple paths and introduce the spatial diversity for the data stream in the channel. It is unlikely that all the paths encounter severe fading at the same time, therefore the MIMO scheme improve the signal liability in a natural wireless environment. In addition, spatial diversity increase the antenna gain further with the space-time coding technique applying to the transmission information.

However, the design complexity of the MIMO detection at the receivers is also increased. Maximum Likelihood(ML) technique is often used for MIMO detection in VLSI implementations. It can provide either hard or soft decoding output estimation . Specifically, hard decoding derive the nearest constellation to the unconstrained estimate from the equalization procedure. Whereas soft decoding computes a posteriori probability(APP) of each possible transmitted symbol and then derives the decoding result using techniques such as turbo decoding. Though exhaustive search is the most straightforward way to calculate the ML result, its complexity increases exponentially along with the increase of number of the transmission antennas and the bits per symbol in the constellations. Recently sphere detector has been shown to reduce the complexity while maintain ML performance and is feasible for VLSI hardware implementation. Unfortunately, though using sphere detector, the design of MIMO SoCs is limited due to the high computational complexity of 
MIMO algorithms and stringent power constraints of MIMO applications. This thesis presents a SoC implementation of the $3 \mathrm{G}$ CDMA receiver that utilizes the MIMO technology. The receiver targets at a MIMO channel with four transmit antennas and four receive antennas. Our chip has a very high level of system integration. It contains all digital processing components of the MIMO CDMA receiver and is able to perform signal equalization, maximum likelihood MIMO detection, and turbo decoding. The novelty of our design also stems from its reconfigurability. The current implementation can be reconfigured in into two decoding modes that handle 16QAM and QPSK modulations, respectively. More modulation schemes can be handled by replacing a ROM in our design with a SRAM and corresponding flash memory programing circuits.

In addition, we perform a thorough analysis to find the best trade-off between system performance and hardware complexity. Our RTL design is implemented with variable whose width can be changed. Fixed-point simulations in MATLAB are used to find out the system performance of various configurations. We change the bit width of a set of variables in both the MATLAB code and RTL descriptions and exam the performance after simulation and accurate hardware complexity in area and power consumption after synthesis. We examine the performance and find out the best design configuration.

Our SoC design strategy achieves a high-performance and low-power MIMO receiver. In particular, the integration of a large number of modules on a single chip not only provides a high computational capacity but also reduces the overhead due to the data transfer among modules. Our MIMO receiver is designed using a $0.18 \mu \mathrm{m}$ CMOS technology. Simulation results have shown that our receiver achieves a $5 \mathrm{Mbps}$ transmission rate at each user's end. The chip core area and average power dissipation are $16.5 \mathrm{~mm}^{2}$ and $557 \mathrm{~mW}$, respectively.

\subsection{Contribution}

This thesis introduces a new idea of implementing the MIMO equalizer, detector and the turbo decoder onto one single chip to improve the overall system performance. We also provide the ability for the chip to use either QPSK modulation scheme or 16QAM modulation scheme according to SNR range. Finally, we describe an optimization scheme to find the trade-off between hardware complexity in terms of silicon area and power consumption and system performance in terms of BER. 


\subsection{Thesis Organization}

The rest of this thesis is organized as follows. Chapter 2 presents the background information on MIMO wireless communication system and MIMO hardware design and a survey of previous related research. Chapter 3 proposes an overview of our MIMO receiver design. Chapter 4 presents the detail RTL design of module equalizer design. The flat channel estimation module design is also presented in Chapter 3. Chapter 5 proposes the detail RTL design of sphere decoder. Chapter 6 proposes the detail RTL design of turbo decoder. Chapter 7 presents our optimization scheme on this design. Chapter 8 presents the chip design result of our MIMO SoC. Chapter 9 summarizes this thesis. 


\section{Chapter 2}

\section{Background and Related Work}

In this chapter, we first introduce the MIMO CDMA communication architecture. We next present the turbo decoding theory mathematically. We then summarize previous MIMO hardware design work.

\subsection{Background}

\subsubsection{MIMO CDMA Communication Architecture}

Fig. 2.1 shows a block diagram of a MIMO CDMA architecture. It has 4 transmit and 4 receive antennas. The raw information source data from several users are encoded using turbo codes. The turbo code blocks are mapped to data symbols according to a certain modulation scheme. Then the symbols are spread using Walsh sequences, which are different for various users. The symbols from multiple users are added together to form the transmitted symbols. The transmitted symbols are distributed to four transmission paths evenly using a demux. The pilot symbols are added into the transmitted symbols at each path and the result signals are multiplied with scrambling codes to increase the overall signal randomness. The scrambled symbols are sent to the transmit antenna. Note that different transmission paths have different pilots and scrambling codes, but use the same encoding symbol creating scheme for all users.

The transmitted signals from all antennas are combined in the wireless channel together with 


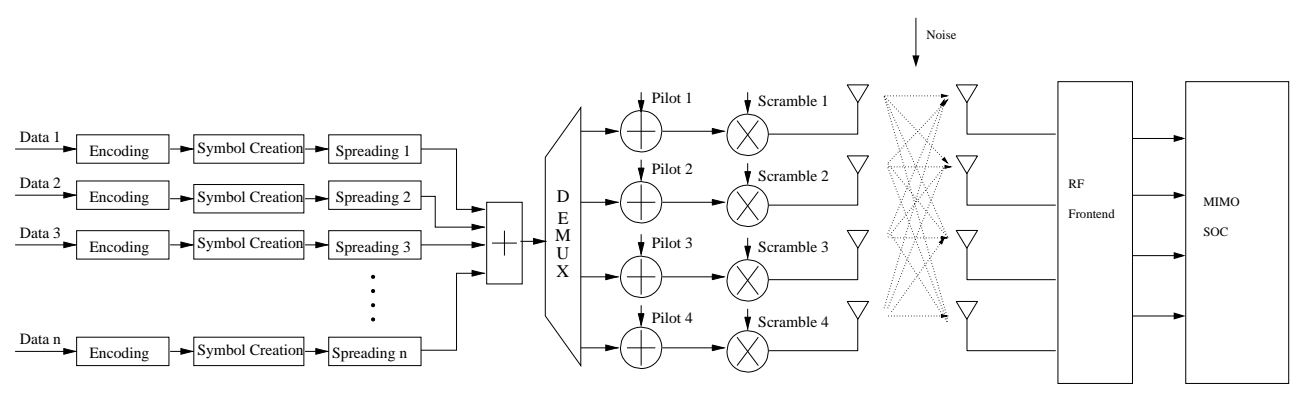

Figure 2.1: A CDMA transmitter diagram.

the background noise which is modeled as addictive white Gaussian noise (AWGN). In addition, the variation of the signal propagation delays through different transmission paths leads to inter-symbol interference, i.e., signals transmitted at different time within a time window $N$ may interfere with each other.

At the receiver side, the continuous signals at the receiver antennas are sampled in a RF front end to produce the received symbols. Spatial diversity is achieved with this multiple antennas architecture at the receiver. If $x_{k, j}$ represents the transmitted symbol $j$ at antenna $k \in\{0,1,2,3\}$ and $N$ is the interference window width, the $i$ th received symbol at receive antenna $m \in\{0,1,2,3\}$, denoted as $r_{m}(i)$, can be described as

$$
r_{m}(i)=\vec{C}_{m} \cdot \vec{x}(i)+n(i)
$$

where $n(i)$ is the AWGN at $i$ th receive antenna, $\vec{C}_{m}$ is the space-time channel vector with a dimension of $4 \times N$, and $\vec{x}$ is a vector consisting of transmitted signals $x_{k, j}, k \in\{0,1,2,3\}, j \in\{i, i-1, \ldots, i-$ $N+1\}$.

The receiver first performs an interference cancellation using adaptive filters. The filter outputs are then despread and sent to a space-time MIMO detector. The detector perform a maximum likelihood estimation to effectively eliminate the white Gaussian noise. Finally, with a soft decoding information from the detector, Turbo decoding can be used to eliminate the noise further to reduce to bit error rate $[4,11,17]$, which is described in following section.

\subsubsection{MIMO Channel Model}

We model the wireless channel as a frequency selective channel with inter-symbol interference. The interference length is set to 16 , which is a typical case for the wireless communication 
environment. The transfer function of the channel is written as following:

$$
H(\omega)=H_{0} \sum_{n=1}^{16} \alpha^{n-1} e^{-j \omega n}+N
$$

where $\alpha$ is a constant smaller than $1, \mathrm{~N}$ denotes the White Gaussian Noise, and $H_{0}$ is a $4 \times 4$ flat channel matrix which is randomly generate by MATLAB. In our design, we set the $\alpha$ value as 0.3 , which is a typical value for the wireless channel. The mean of the White Gaussian Noise is zero and the variance is based on the SNR level.

\subsubsection{Turbo Decoding}

This section describe the turbo decoding technique mathematically. A simplification scheme is applied to make the hardware implementation feasible. Let $\vec{d}=\left(d_{1}, \ldots, d_{N}\right)$ be a binary information bits sequence, where $\mathrm{N}$ is the frame size. Two outputs are generated after encoding: systematic information bits $\vec{x}^{s}=\left(x_{1}^{s}, \ldots, x_{N}^{s}\right)=\vec{d}$ and partity information bits $\vec{x}^{p}=\left(x_{1}^{p}, \ldots, x_{N}^{p}\right)$. After a noise channel, $\vec{y}^{s}$ and $\vec{y}^{p}$ are the corresponding received sequences and they are denoted as $\vec{y}=\left(\vec{y}^{s}, \vec{y}^{p}\right)$. Maximum a posteriori(MAP) algorithm is often used in the turbo decoding process. The goal of a MAP Decoder is to calculate the log-likelihood ratio(LLR) of the received $\vec{y}$ in order to estimate the info bits:

$$
\operatorname{LLR}\left(d_{k}\right)=\log \frac{P\left(d_{k}=+1 \mid \vec{y}\right)}{P\left(d_{k}=-1 \mid \vec{y}\right)}
$$

where $d_{k}$ is the time index of $\vec{d}[3]$.

Let the state of the encoder at time $\mathrm{k}$ be $m$ and at time $k-1$ be $m / . m$ can take on values between 0 and $2^{M}-1$ where $M$ is the number of memory elements in the RSC encoder. We can rewrite Equation (2.3) using Bayes rule as

$$
L L R\left(d_{k}\right)=\log \frac{\sum_{m} \sum_{m^{\prime}} \alpha_{k-1}(m \prime) \cdot \beta_{k}(m) \cdot \gamma_{1}\left(y_{k}, m, m^{\prime}\right)}{\sum_{m} \sum_{m^{\prime}} \alpha_{k-1}\left(m^{\prime}\right) \cdot \beta_{k}(m) \cdot \gamma_{0}\left(y_{k}, m, m^{\prime}\right)}
$$

where $\alpha$, called the forward recursion metric is the probability of being in state $m$ at time $k$ with the received sequence $\left(\vec{y}_{1}, \ldots, \vec{y}_{k}\right), \beta$, called the backward metric is the probability of generating the received sequence $\left(\vec{y}_{k+1}, \ldots, \vec{y}_{N}\right)$ from state $m$ at time $k$, and $\gamma$, called the branch transition metric is 
the probability of transiting from state $m /$ to state $m$ [1]. They are mathematically defined as:

$$
\begin{array}{r}
\alpha_{k}(m)=\sum_{m \prime} \sum_{i=0}^{1} \gamma_{i}\left(y_{k}, m, m^{\prime}\right) \cdot \alpha_{k-1}\left(m^{\prime}\right), \\
\alpha_{0}(0)=1, \alpha_{0}(m \neq 0)=0 ; \\
\beta_{k}(m)=\sum_{m \prime} \sum_{i=0}^{1} \gamma_{i}\left(y_{k}, m, m \prime\right) \cdot \beta_{k+1}\left(m^{\prime}\right), \\
\beta_{N}(0)=1, \beta_{N}(m \neq 0)=0 ; \\
\gamma_{i}\left(y_{k}, m, m^{\prime}\right)=P\left(d_{k}=i \mid m, m^{\prime}\right) \cdot P\left(y_{k}^{s} \mid d_{k}=i\right) . \\
P\left(y_{k}^{p} \mid d_{k}=i\right) \cdot P\left(m \mid m^{\prime}\right) .
\end{array}
$$

To avoid the complexity of huge multiplications in Equation (2.5) (2.7) and (2.9), we computes the logarithms of the $\alpha, \beta$ and $\gamma$ instead so that we can use additive operation to implement the above three equations:

$$
\begin{array}{r}
\bar{\alpha}_{k}(m)=\ln \alpha_{k}(m), \\
\bar{\beta}_{k}(m)=\ln \beta_{k}(m), \\
\bar{\gamma}_{i}\left(y_{k}, m, m \prime\right)=\ln \gamma_{i}\left(y_{k}, m, m \prime\right) .
\end{array}
$$

To reduce the hardware complexity, the Jacobian logarithm is used in the calculation with approximation:

$$
\ln \left(e^{u_{1}}+\ldots+e^{u_{n}}\right)=u_{M}+\ln \left(1+\sum_{i \neq M} e^{u_{i}-u_{M}}\right), u_{M}=\max \left(u_{i}\right) .
$$

The second term can be implemented with a one-dimensional look-up table.

Now we can rewrite the equations of forward metrics and backward metrics as following:

$$
\begin{aligned}
& \bar{\alpha}_{k}(m)=\overline{\max }_{(m \prime, i)}\left(\bar{\gamma}_{i}\left(y_{k}, m, m \prime\right)+\bar{\alpha}_{k-1}(m \prime)\right), \\
& \bar{\beta}_{k}(m)=\overline{\max }_{(m \prime, i)}\left(\bar{\gamma}_{i}\left(y_{k}, m, m \prime\right)+\bar{\beta}_{k+1}(m \prime)\right),
\end{aligned}
$$

where $\overline{\max }$ is the notation for the function introduced in Equation(2.13)

$$
\overline{\max }\left(u_{i}\right)=u_{M}+\ln \left(1+\sum_{i \neq M} e^{u_{i}-u_{M}}\right), u_{M}=\max \left(u_{i}\right)
$$


Then Equation (2.4) can be simplified as shown in [13]:

$$
\begin{aligned}
L L R\left(d_{k}\right) & =\overline{\max }_{m,(m \prime)}\left(\bar{\alpha}_{k-1}(m \prime)+\bar{\beta}_{k}(m)+\bar{\gamma}_{1}\left(y_{k}, m, m^{\prime}\right)\right) \\
& -\overline{\max }_{m,(m \prime)}\left(\bar{\alpha}_{k-1}(m \prime)+\bar{\beta}_{k}(m)+\bar{\gamma}_{0}\left(y_{k}, m, m \prime\right)\right)
\end{aligned}
$$

The branch metrics are calculated with [16]

$$
\bar{\gamma}_{k}\left(y_{k}, m, m \prime\right)=\frac{1}{2}\left[L_{i n}^{e}\left(y_{k}\right) y_{k}+L_{c} y_{k}^{s} d_{k}+L_{c} y_{k}^{p} x_{k}^{p}\right],
$$

where $L_{i n}^{e}\left(y_{k}\right)$ is the a priori information calculated by the other decoder and $L_{c}=4 E_{c} / N_{0}$. In a White Gaussian Channel, $N_{0}$ is the noise spectral density and $E_{c}$ is the energy per coded bit.

\subsection{Previous MIMO Receiver Design}

Thousands of papers have been published in the area of theoretical MIMO systems design and optimization since the MIMO technique was first introduced about a decade ago. however, few hardware implementation of MIMO system in FPGA or ASIC was reported since the large complexity of the algorithms. In addition, the tight power consumption constrain for real application make it more difficult. We reviewed several attempts which have been made to implement MIMO based systems. In particular, the Bell Lab Layered space-time architecture is the first MIMO architectural design [8]. Early hardware prototypes of MIMO implementations are based on DSP microprocessors or FPGAs $[10,12,15]$. These early designs dissipate prohibitively high power and are therefore impractical for mobile applications. The ASIC implementation of a MIMO receiver has been developed by Bell Labs, Lucent Technologies, targeting at the high speed downlink packet access applications for 3G networks [9]. This chip can only handle QPSK modulation for $4 \times 4$ MIMO configuration, i.e., four transmit and receive antennas, however. Airgo Networks Inc., has also announced its MIMO chip set, targeting at the next-generation broadband wireless LAN applications[18]. Other resent ASIC implementation of MIMO receiver target at improving throughput at high SNR while have low BER at SNR range around $10 \mathrm{~dB}[5,6]$. To our knowledge, however, no single chip implementation of MIMO receiver has been reported in the literature that consists of both adaptive filtering and Turbo decoding. 


\section{Chapter 3}

\section{Overview of MIMO Receiver Design}

Fig. 3.1 shows the overall system architecture with five key components. The input buffer serves as the temporary storage for the received symbols from the RF front end. The buffer should be large enough to store doubles the length of processing symbols since the incoming data is continuous without interrupt. The equalization module applies the adaptive filtering technique to remove the inter-symbol interference and spatial correlation among antennas. It also despreads the equalized symbols. The flat channel estimation module uses the equalization results to estimate the channel matrix. The sphere decoder produces a posteriori probabilities (APP) of potential transmitted symbols. The APP results are then used by the turbo decoder to compute the user data. The following chapters describe the detailed implementations of the equalization module, the sphere decoder, and the turbo decoder.

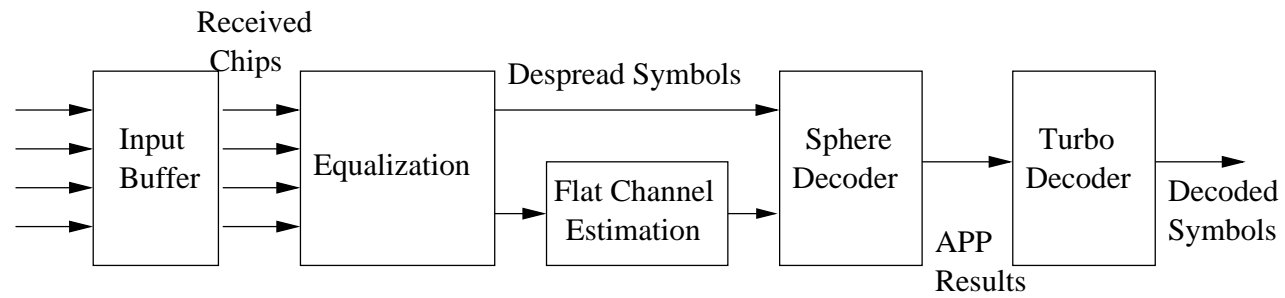

Figure 3.1: proposed MIMO receiver diagram. 


\section{Chapter 4}

\section{RTL Design of Equalization and Flat}

\section{Channel Estimation Module}

\subsection{Equalization Module}

The equalization module consists of four equalizers, deriving the transmitted symbols from four transmit antennas, respectively. The difference among them is that different pilots and scrambling codes are used. Fig. 4.1 shows the block diagram of one equalizer. It contains four finite impulse response (FIR) filters, one for each receive antenna. The filter lengths are set to 16 to handle inter-symbol interference that spans across 16 consecutive symbols. The filter outputs are added together. The summation result is despread to generate the equalized symbols.

We adopt the correlation-based scheme in [9] to generate the coefficients for the FIR filters. Specifically, two correlator modules are designed. The input correlator contains four submodules, each of which performs the correlation of one distinct input symbol sequence and a special pilot sequence, called C-SS pilot. The C-SS pilot is the conjugate of the spread and scrambled pilot. The output correlator correlates the summation of four FIR outputs and the C-SS pilot. The result derived by the output correlator is compared with the expected amplitude. The difference, denoted as the normalized least mean square (NLMS) error, is then used in conjunction with the outputs from the input correlator to update the coefficients of the FIR filters. 


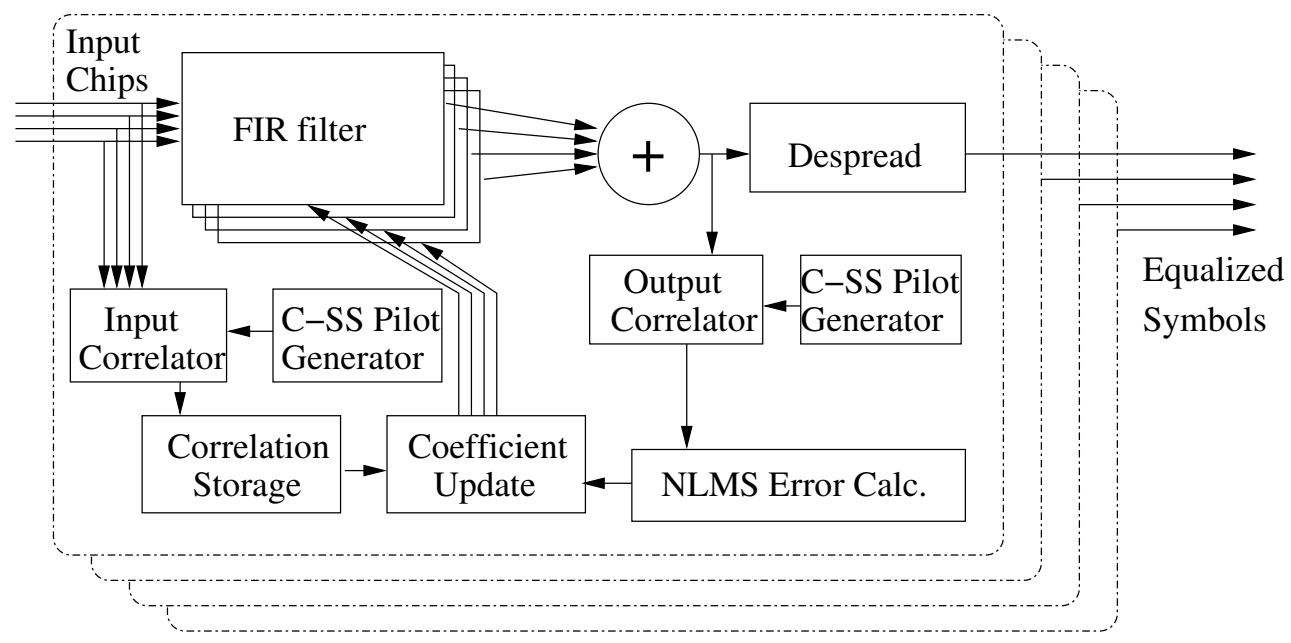

Figure 4.1: Equalizer diagram.

\subsubsection{FIR Filter}

The detail RTL implementation is shown in Figure 4.2. It constrains four complex multiply and accumulate units, each unit is connected to the received data from one antenna and the coefficient value. The accumulated values are added up to carry out the output of the FIR filter. The coefficient values of the FIR filter are adaptively updated by the coefficient update module according to the correlator result both on the input and the output. Therefore inter-symbol interference is greatly reduced after the FIR filter.

\subsubsection{Correlator}

The correlator is implemented by using four multiply and accumulator units with the input of pilot and the received data. The block diagram is shown in Figure 4.3. The output of the correlator is written into four correlation memories, each for one transmitter antenna.

\subsubsection{C-SS Pilot Generator}

The pilot signal is based on the state generated by a Linear Feedback Shift Register (LFSR), as shown in Figure 4.4. The length of the LFSR is set to 16 to have a long pseudorandom sequence. The LFSR polynomial is:

$$
x^{15}+x^{13}+x^{4}+1
$$




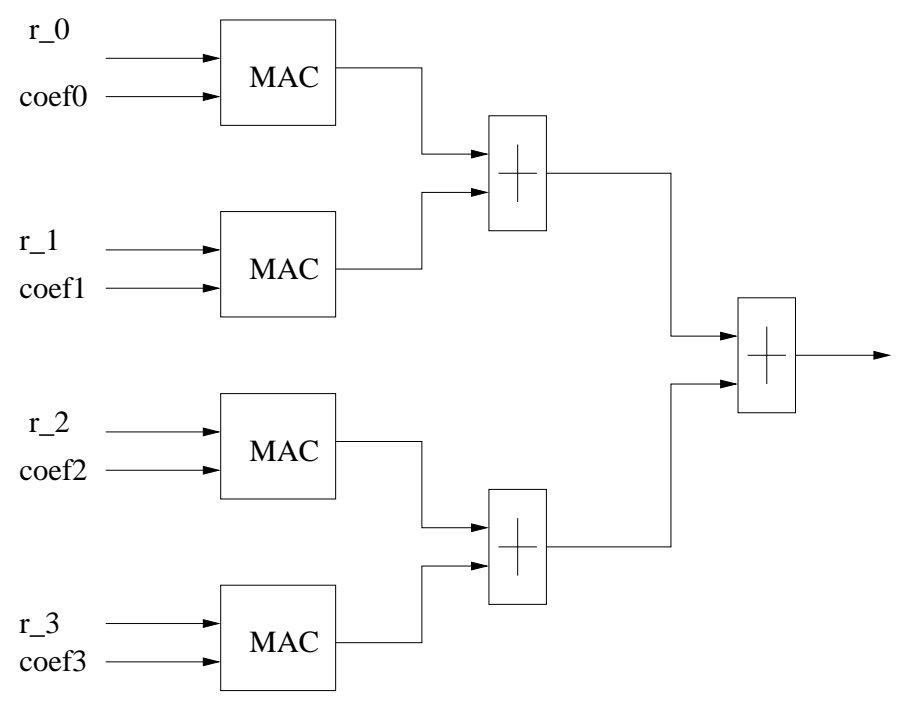

Figure 4.2: FIR block diagram.

The scramble code is generated with the LFSR with the similar fashion. The 16 bits complex C-SS pilot sequence is then generated by spreading and scrambling the pilot following by changing the sign of the imaginary part.

\subsubsection{NLMS Error Calculation and FIR Tap Updating}

To calculate the NLMS errors, we subtract the input correlator value from and configurable complex constant, which is set to $0.5+0 i$ in our design. The result of the subtraction is multiplied with a normalized factor 1/16. The tap of the FIR filter is updated by the circuit as shown in Figure 4.5. The NLMS error and the correlator input is multiplied. The new weight of the FIR filter is the sum of the previous weight and the multiply result. It should be noted we have four copies of this block to generate four sets of taps in each equalizer.

\subsection{Flat Channel Estimation Module}

The flat channel estimation module use the spreaded pilot signals to estimate the $4 \times 4$ channel matrix. The pilot signal vector which is generated from a PN sequence is orthogonal to the information bits. Therefore the following estimation can be considered reliable. 


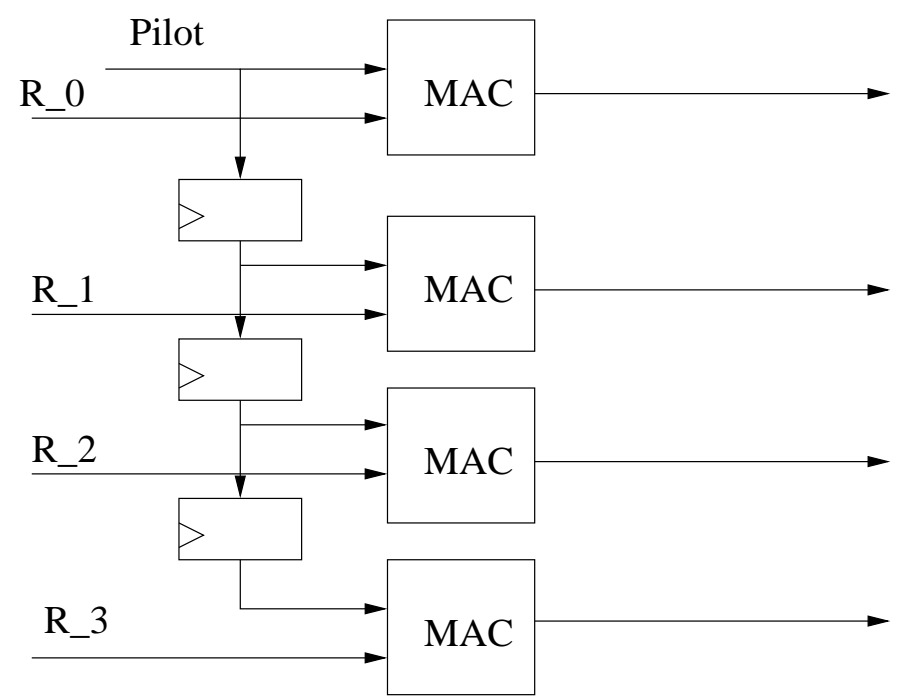

Figure 4.3: Correlator block diagram.

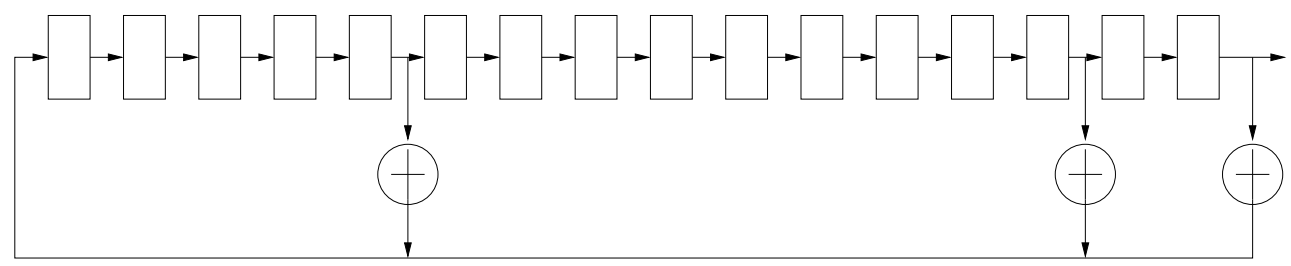

Figure 4.4: LFSR block diagram.

$$
H_{i, j}=\sum r_{i} * \operatorname{conj}(\operatorname{pilot}(j)) / \sum \operatorname{pilot}(j) * \operatorname{conj}(\operatorname{pilot}(j))
$$

where $i$ is the receive antenna index and $\mathrm{j}$ is the transmit antenna index, pilot is the spreaded pilot vector. It should be noted that the amplitude of the pilot should be careful chosen so that the denominator of above equation to be the power of 2 to avoid division operation in hardware.

\subsection{Verification Procedure}

We derive a system model in MATLAB for the equalizer and the flat channel estimation modules. Random user data packages and a random flat matrix channel model are generated by a MATLAB program. Then we feed the output of the MATLAB transmitter to the input of the equalizer 


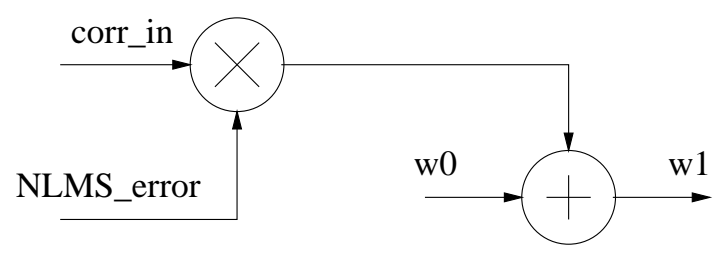

Figure 4.5: Weight update block diagram.

and flat channel estimation RTL models to perform a Verilog simulation. The simulation will write out the despreaded data stream to an output file. We compare the data in the output file with the output from fixed-point MATLAB simulation. We also compare the estimated flat channel matrix by Verilog with the matrix by MATLAB. The Verilog design is considered correct when the two outputs match. 


\section{Chapter 5}

\section{RTL Design of Sphere Decoder Module}

\subsection{Overview of Sphere Decoder Module Design}

The equalization module is not effective in reducing the white Gaussian background noise in the communication channel. As a result, its output symbols $\vec{y}$ unlikely match any constellations. If the entire effect of the channel and equalization procedure is modeled as a flat-channel matrix $\hat{H}$, the transmitted symbol vector $\vec{s}^{*}$ should satisfy $\vec{y}=\hat{H} \vec{s}^{*}$. In practice, a flat channel matrix estimation $H$ is used to approximate $\hat{H}$. The transmitted symbol vector $\vec{s}=\left\{s_{0}, s_{1}, s_{2}, s_{3}\right\}$ that minimizes the cost function $J(\vec{s})=\|H \vec{s}-\vec{y}\|^{2}$ can be used as the estimation for $\vec{s}^{*}$.

Our MIMO receiver targets at the configuration of 4 transmit antennas and 16 QAM modulation. Consequently, there exist $16^{4}=65,536$ different symbol vectors, making it impractical to derive the $\vec{s}$ that minimizes $J(\vec{s})$ by calculating the cost functions of all possible symbol vectors. We implement a technique called sphere decoding to simplify the search for the minimal $J(\vec{s})$. The basic principle of sphere decoding is that the symbol vector $\vec{s}$ which results in a small $J(\vec{s})=\|H \vec{s}-\vec{y}\|^{2}$ is likely to have a small $\left\|\vec{s}-H^{-1} \vec{y}\right\|^{2}$. Consequently, we only calculate $J(s)$ for a limited number of $\vec{s}$ around the location $H^{-1} \vec{y}$. 


\subsection{Sphere Decoding Searching Procedure}

Our sphere decoding procedure can be explained by Fig. 5.1. The black dots represent the symbol vectors. The unconstrained estimation $s^{\prime}=H^{-1} \vec{y}$ is first calculated. Using $s^{\prime}$ as the center, a circle with a gradually increasing radius is created. A search path can be formed by placing the black dots, i.e., symbol vectors, in the order that they are crossed by the circle. The number of symbols on the path is selected based on the receiver throughput requirement. In Fig. 5.1, the path is represented in dotted lines and numbers is the searching steps. Apparently, different $s^{\prime}$ may have different search paths. We partition the symbol vector space into several regions. All $s^{\prime}$ in a single region share the same path. A memory module is designed as a look-up table to store all paths so that, whenever an $s^{\prime}$ is derived, the corresponding search path can be applied.

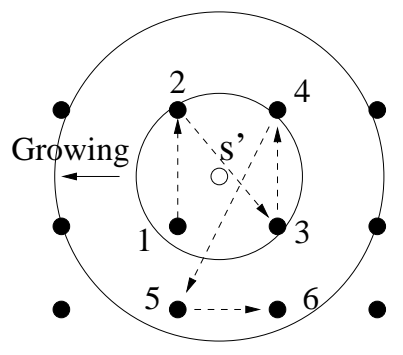

Figure 5.1: Sphere decoder algorithm illustration.

\subsection{Block Diagram of the Sphere Decoder}

The block diagram of the sphere decoder is shown in Fig. 5.2. It can be divided into two major parts. The first part includes the decomposition of channel matrix and calculation of the unconstrained transmitted symbols [7]. The second part performs a search procedure that derives the minimum cost function. In particular, a matrix computation block computes the symbol cost function and a matrix enumeration block determines the next candidate symbol along the search path. Both matrix computation and enumeration blocks contain two identical datapaths that operate in parallel for high throughput. All search paths are stored in the path look-up memory (PLM). In our design, the PLM is a ROM that contains paths created for QPSK and 16QAM modulations. Other modulation schemes can be handled by changing the PLM contents or converting the ROM 
into a programmable flash memory. A book-keeping module records the cost function values of all symbols visited during the search. It also computes the log-likelihood ratios (LLRs) for each equalizer output $\vec{y}$ as follows.

$$
\ln \left(\frac{P\left(b_{j}=1 \mid \vec{y}\right)}{P\left(b_{j}=0 \mid \vec{y}\right)}\right) \approx \min _{b_{j}(\vec{s})=0, \forall \vec{s} \in S P} J(\vec{s})-\min _{b_{j}(\vec{s})=1, \forall \vec{s} \in S P} J(\vec{s}),
$$

where $b_{j}$ is the bit $j$ of the transmitted symbol and $S P$ is the search path. The transmitted symbols can then be estimated using the LLRs.

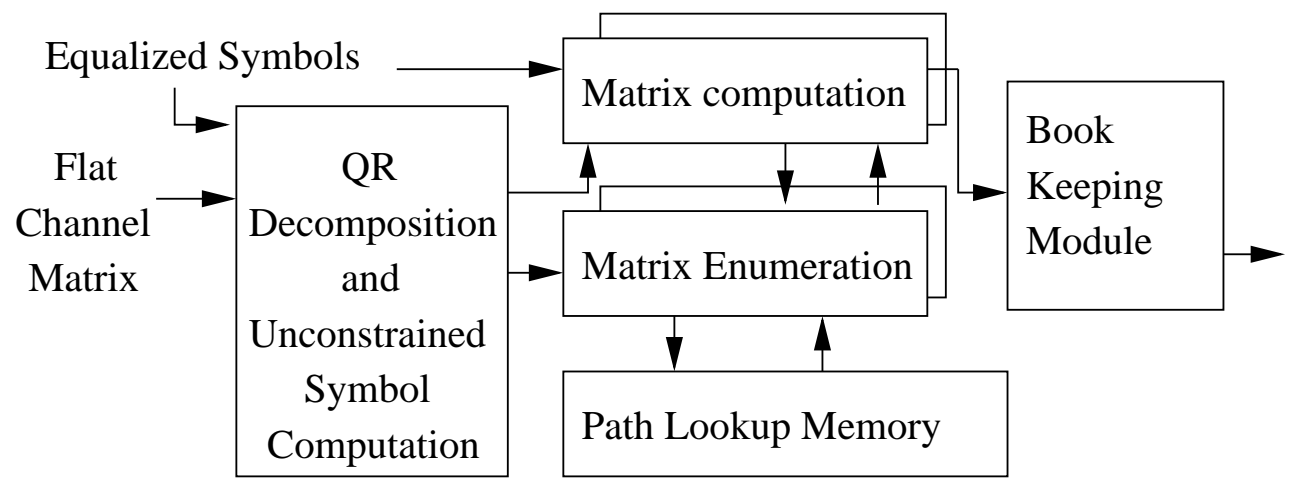

Figure 5.2: Sphere decoder diagram.

\subsubsection{QR Decomposition and Unconstrained Symbol Computation}

The goal of QR decomposition is to derive the decomposition of the channel matrix $H$ into an orthogonal and triangular matrix, as defined in the following equation:

$$
H=Q * R
$$

where $\mathrm{Q}$ is an orthogonal matrix and $\mathrm{R}$ is an upper triangular matrix of the same dimension as $H$. To avoid square-root and division operations, we can represent the matrix $R$ by[7]

$$
r_{i j}=\frac{p_{i j}}{\sqrt{k_{i}}}
$$

Now the channel matrix $H$ can be expressed in:

$$
H=Q * K^{-1} P
$$

where $K^{-1}$ is an diagonal matrix given by the real valued weights $1 / k_{i j}$, and the upper triangular matrix $P$ is calculated by the scaled Givens rotation of $H$. 


\subsubsection{Matrix computation}

The matrix computation module recursively calculate the cost function fro the candidate symbol $s_{p l m}$ from the matrix enumeration module. As shown in Figure 5.3, the partial Euclidean distance (PED) unit is used to calculate the PED of the two symbols on the constellation diagram using the squared absolute subtraction value of $s^{\prime}$ and $s_{p l m}$. The PED is added with the previous cost function and then subtract from the multiplication of the inversed matrix $\mathrm{P}$ and the received symbol from the equalizer. Four copies of this circuits are running parallel to increase the throughput of the sphere decoder.

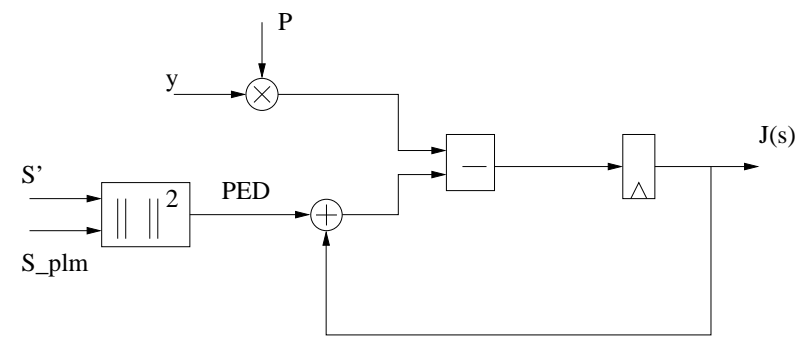

Figure 5.3: Matrix computation block diagram.

\subsubsection{Matrix Enumeration}

The matrix enumeration enumerate the next candidate symbol on the constellation diagram along the sphere search path. The block diagram of this module is shown in Figure 5.4. It first calculate the PED of all symbols on the searching path from the PLM using the PED from the matrix computation module. Then a minimum search algorithm is performed to the cached PED values to select four preferred children from the candidate symbols, one for each transmitter. The enumeration output module sent the four children symbol to the matrix computation module the calculate the cost function and LLR.

\subsubsection{Book Keeping}

The book keeping module records the cost function values of all symbols $\vec{s}$ during the search. The detail block diagram of the recording circuit is shown in Figure 5.5. A subtraction operation is performed to current cost function input and the minimum cost function, the sign of the result 


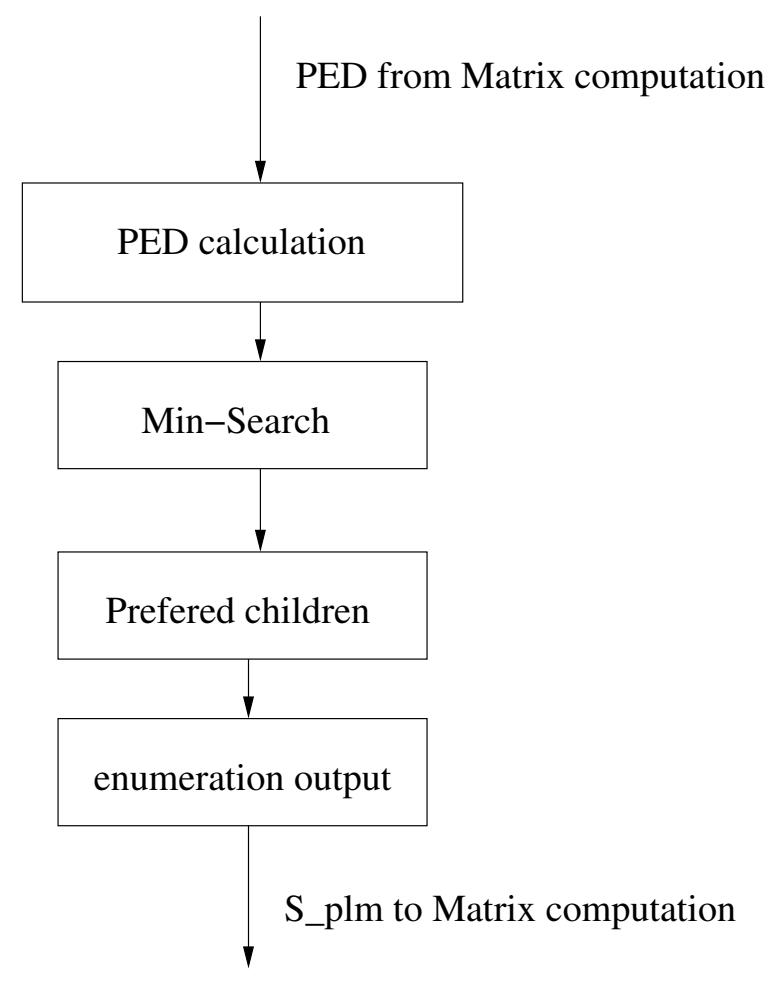

Figure 5.4: Matrix enumeration block diagram.

is used to select the smaller value via a MUX. There are two identical recording circuits in this module, both for the bit as "1" and for bit as "0". Finally, this module also computes the LLR for each output using Equation (5.1).

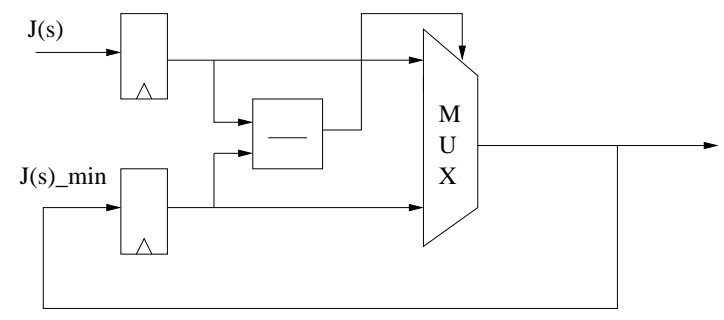

Figure 5.5: Book keeping recording diagram. 


\subsection{Verification Procedure}

We derive a system model in MATLAB for the sphere decoder and run the fixed-point simulation with random user data. The LLR output from the MATLAB is written to a file. Then we run the simulation with Verilog use the same input as that in the MATLAB. We dump out the output from the Verilog simulation and compare this file with the MATLAB result file.

\section{Acknowledgement}

I would like to thank to Dr. Zhengtao Yu for his effort on the first design of the QR decomposition and unconstrained symbol computation module. His contribution on the module design make this work possible. 


\section{Chapter 6}

\section{RTL Design of Turbo Decoder}

\subsection{Overview of Turbo Decoder Design}

Our Turbo decoder estimate the transmitted data using the LLRs from the sphere decoder and further reduces the communication bit error rate. Its block diagram is shown in Fig. 6.1. It contains a datapath loop since Turbo decoding algorithm performs probability propagation iteratively in the Trellis graph defined by the encoding procedure. In particular, it first derives the branch transition metric $\gamma$ based on the data from the sphere decoder. It then calculates the forward recursion metric $\alpha$ and the backward recursion metric $\beta$. The values of $\alpha, \beta$, and $\gamma$ are updated iteratively and used to derive the decoding results. Although Turbo decoding algorithm consists two decoding processes that operate interchangeably, we implement only one decoder and use it in a time-multiplexing manner to reduce the chip area. We pipeline the datapath for high throughput. Furthermore, we apply the sliding window scheme [2] that limits the length of probability propagation to 16 Trellis columns for memory reduction. In the sliding window scheme, the state probabilities or branch transition metrics in three consecutive windows are calculated simultaneously using the $\alpha, \beta, \gamma$, and pre- $\beta$ blocks. Total 11 rounds of iterations are performed. 


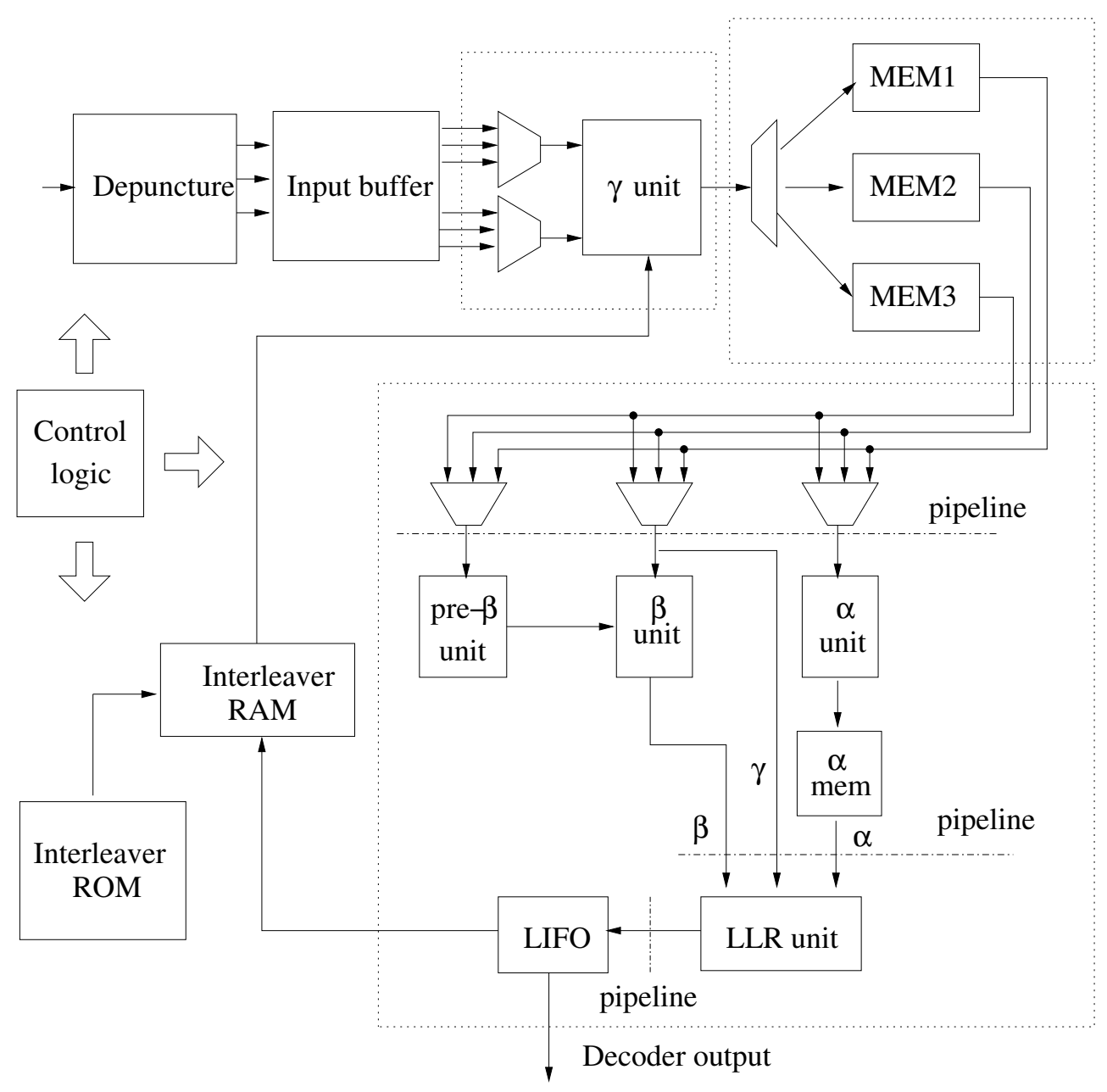

Figure 6.1: Turbo decoder.

\subsection{Detail Data Flow in the Turbo Decoder}

The LLR is sent to the input buffers after depunctured. Two sets of buffers are used so that we can store the next data block when processing the current block. A mux is followed to select the systematic info and parity info bits from the two buffers. These two info bits are sent to the $\gamma$ unit along with the interleavered $L_{e}$ to calculate the branch metric. Three memory are used to store the $\gamma$ of the adjacent 3 sliding window blocks. For convenience, we note it as $\gamma_{1}, \gamma_{2}$ and $\gamma_{3}$. Three three-to-one MUX are used to select the proper $\gamma$ for the calculation of pre- $\beta, \beta$ and $\alpha$. pre- $\beta$ unit is used to carry out the initial value for the $\beta$ unit. We don't store the pre- $\beta$ and $\beta$ value according to the sliding window algorithm. Therefore, a large size of hardware resource is saved. However, we 
use a memory to store the $\alpha$ value of each sliding block. We must point out that the $\alpha$ value is read in a reverse order from the $\alpha$ memory. The $\beta, \alpha$ and $\gamma$ value of the same sliding block are sent to the LLR unit to calculate the LLR and $L_{e}$ value. Since the order of the LLR/ $L_{e}$ is reversed, a LIFO is implemented to carry out the $\mathrm{LLR} / L_{e}$ in timing index order. The value of $L_{e}$ will be sent to the $\gamma$ unit after interleaver/deinterleaver.

\subsection{The Operation of the Sliding Windows}

Phase 1: the gamma $\left(\gamma_{1}\right)$ of the first block is calculated and stored in MEM1

Phase 2: the gamma $\left(\gamma_{2}\right)$ of the second block is calculated and stored in MEM2

Phase 3: the gamma $\left(\gamma_{3}\right)$ of the third block is calculated and stored in MEM3.

pre- $\beta$ is calculated with $\gamma_{2}$.

$\alpha$ is calculated with $\gamma_{1}$.

Phase 4: the gamma $\left(\gamma_{1}\right)$ of the 4th block is calculated and stored in RAM1

pre- $\beta$ is calculated with $\gamma_{3}$.

$\alpha$ is calculated with $\gamma_{2}$.

$\beta$ is calculated with the initial value of the final value of

pre- $\beta$ at Phase3. Gamma1 is used in the calculation of $\beta$.

LLR of the first sliding block is ready to be calculated at this Phase

Phase 5: the operation at Phase 4 is repeated while the role of the MEM is shifted.

\subsection{Detail Sub-module Design}

\subsubsection{Depuncture}

When we encode the data bits at the transmitter, we puncture the data stream from rate $1 / 3$ to $1 / 2$ by deleting the odd bits of the first parity block and the even bits of the second parity block. At the receiver side, we need to do an inverse operation to recover the data rate from $1 / 2$ to $1 / 3$ so that we can perform the turbo decoding with two parity blocks. This operation is called depuncture. It is performed by insert zeros to the odd bits of the first parity block and the even bits of the second parity block. In the RTL design, we use a 1-bit counter to counter the index of the parity block. The counter start when first LLR bit is arrived. When the counter bit is a "zero", we send the parity bit to 
the first parity output and send a "zero" to the second parity output; similarly when the counter bit is a "one", we send the parity bit to the second parity output and a "zero" to the first parity output.

\subsubsection{Transition Metric Unit}

The transition metric is calculated with the Equation (2.19). In the RTL implementation, we rewrite the above equation as:

$$
\bar{\gamma}_{k}\left(y_{k}, m, m^{\prime}\right)=\frac{L_{c}}{2}\left[\frac{L_{i n}^{e}}{L_{c}}\left(y_{k}\right) y_{k}+y_{k}^{s} d_{k}+y_{k}^{p} x_{k}^{p}\right]
$$

We choose a $L_{c}$ value as 2, which is suitable for a various range of SNR and the circuit is largely simplified. According to the state transition of the turbo encoder, we have four $\gamma$ metrics for four different transitions, as listed in Table 6.1.

Table 6.1: Transition metric calculation

\begin{tabular}{|c|c|c|}
\hline transition metric index & $d_{k}$ & $x_{k}^{p}$ \\
\hline$\gamma_{0}$ & -1 & -1 \\
\hline$\gamma_{1}$ & -1 & +1 \\
\hline$\gamma_{2}$ & +1 & -1 \\
\hline$\gamma_{3}$ & +1 & +1 \\
\hline
\end{tabular}

\subsubsection{Forward and Backward Metric Unit}

The forward and backward metric calculation is base on Equation (2.14) and Equation (2.15). Since we have four states in the turbo encoder, we will calculate four sets of forward and backward metrics. Each set of metric use the corresponding transition metric. The RTL implementation of one forward metric unit is shown in Figure 6.2. The two version of current $\alpha$ and $\gamma$ are summed up. The first version output a "-1" bit and the second version output a "+1" bit according to the turbo encoder scheme. The two sum will be sent to a $\overline{\max }$ operation circuit, which is explained in the following. A subtraction operation is applied to the two sums and the result is sent the absolute block. The absolute block will output the absolute value of the subtraction and then look up for the error correction value in a small Look-Up Table. The sign bit of the subtraction is used to select the larger value of the sum via a Mux. The larger value will be summed up with the correction term to carry out the temporary next $\alpha$ value. A normalization is applied to the temporary next $\alpha$ 
value before output the normalized next $\alpha$ value. The normalization is performed by subtract a fixed normalization value either when there is a normalization request from the input or the temporary $\alpha$ value is larger than a threshold. There is also a normalization request output from this module to send request to other metric calculation module once a normalization is required. The output of the $\alpha$ value is registered.

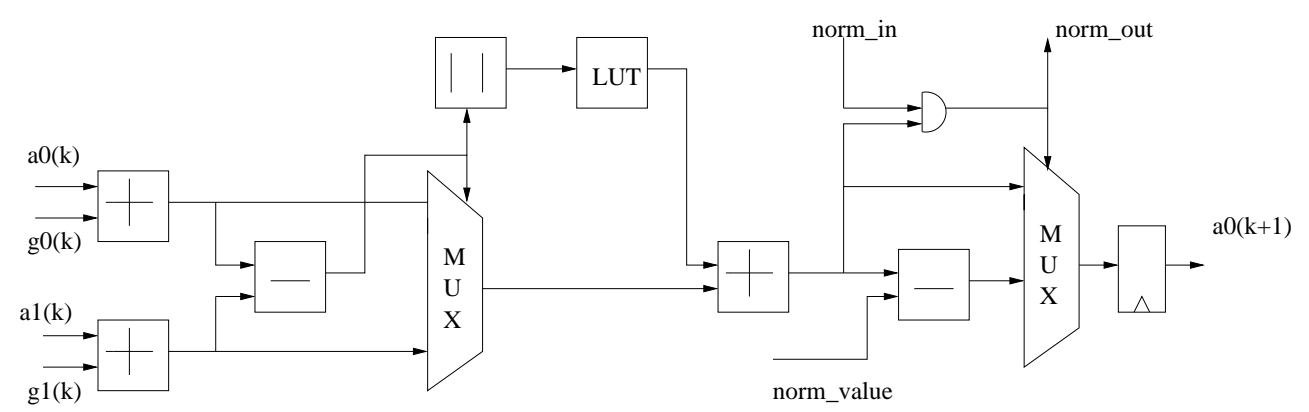

Figure 6.2: forward and backward metric block diagram.

The block diagram for the backward and pre-backward metric calculation is identical to the forward metric excepting the input index of the transition metric and the recursion direction.

\subsubsection{LLR Unit}

The LLR calculation is base on Equation (2.18). The RTL implementation block diagram is shown in Figure 6.3. Three $\overline{\max }$ blocks are used to calculate the log-likelihood of the bit as " $+1 "$. The detail implementation of the $\overline{\max }$ block is part of the circuit shown in Figure 6.2. The LLR unit use another similar circuit to calculate the log-likelihood of the bit as "-1". The final LLR value is calculate by subtract the bit "-1" log-likelihood from the bit" +1 " log-likelihood. It should be noted that the input metrics for the adder should be carefully chosen according to the state transfer scheme in the turbo encoder.

\subsubsection{Interleaving ROM and RAM}

The interleaver ROM is used to generate the interleaved address for the interleaver RAM. Since the block size of the turbo decoder is set to 1024 , both the address width of the interleaver ROM and RAM are 10 bits. The data in the ROM is also 10 bits and generated randomly by software 
program. In even iterations, the $L_{e}$ value from the LIFO are written sequentially and read with the interleaved addresses, while in odd iterations, the interleaved addresses are used for writing and the sequential address for reading.

\subsubsection{Control Logic}

The control logic unit handles the interleaver/deinterleaver of the $L_{e} /$ input info, the window sliding and the iterations. The RTL implement a finite state machine as shown in Figure 6.4.

The detail implementation of each state is listed as in following:

State Initial: Initial state after reset. reset all counters.

State block_start: Start to decode a block. reset iteration counter (iter_cnt).

State iter_start: Start a new decoding iteration. reset sliding window counter (sw_cnt). reset LLR input buffer address pointer(addr_bf). set transition metric memory address pointer(addr_g) to be increase(inc).

State sw_start: Start a new sliding window process. reset bit counter (counter). if signal inc is high, set addr_g to 0 , else set to 15 .

State sw_process: Sliding windows in process. increase addr_bf and counter by one. increase of decrease addr_g according to signal inc.

State sw_end: End of current sliding window. increase sw_cnt by one. flip signal inc.

State iter_end: End of current iteration. increase iter_cnt by one.

State block_end: End of current block. 


\subsection{Verification Procedure}

We derive a system model in MATLAB for the turbo encoder and decoder. Random user data packages are generated and the output data from the encoder is written into a file. When we run the Verilog simulation of turbo decoder, we read in this file as the input of the turbo decoder RTL design. The simulation will also write out the decoded data stream to an output file. We compare the data in the output file with the output from fixed-point MATLAB simulation of the turbo decoder. The Verilog design is modified until the two outputs match. 


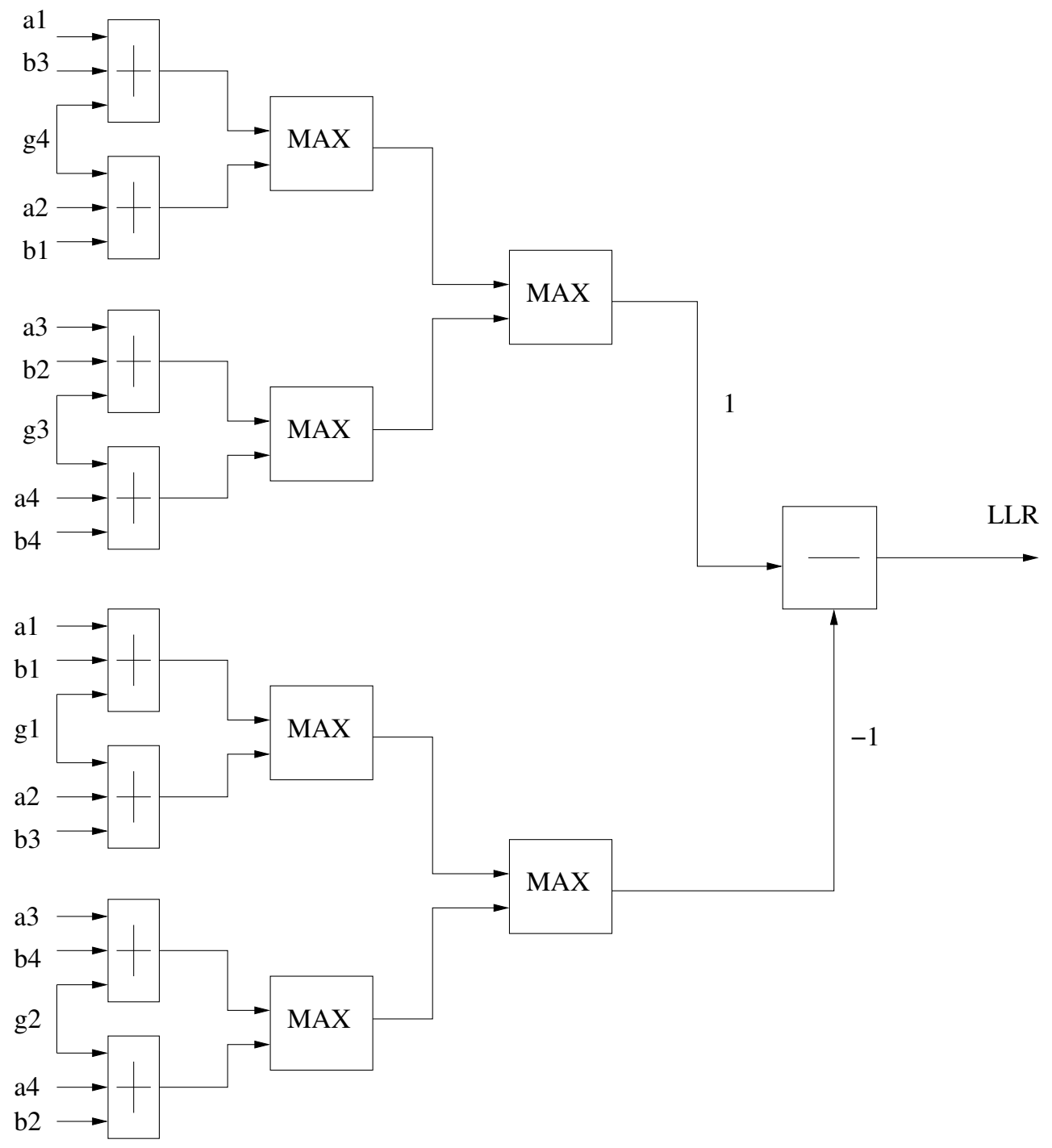

Figure 6.3: LLR calculation block diagram. 


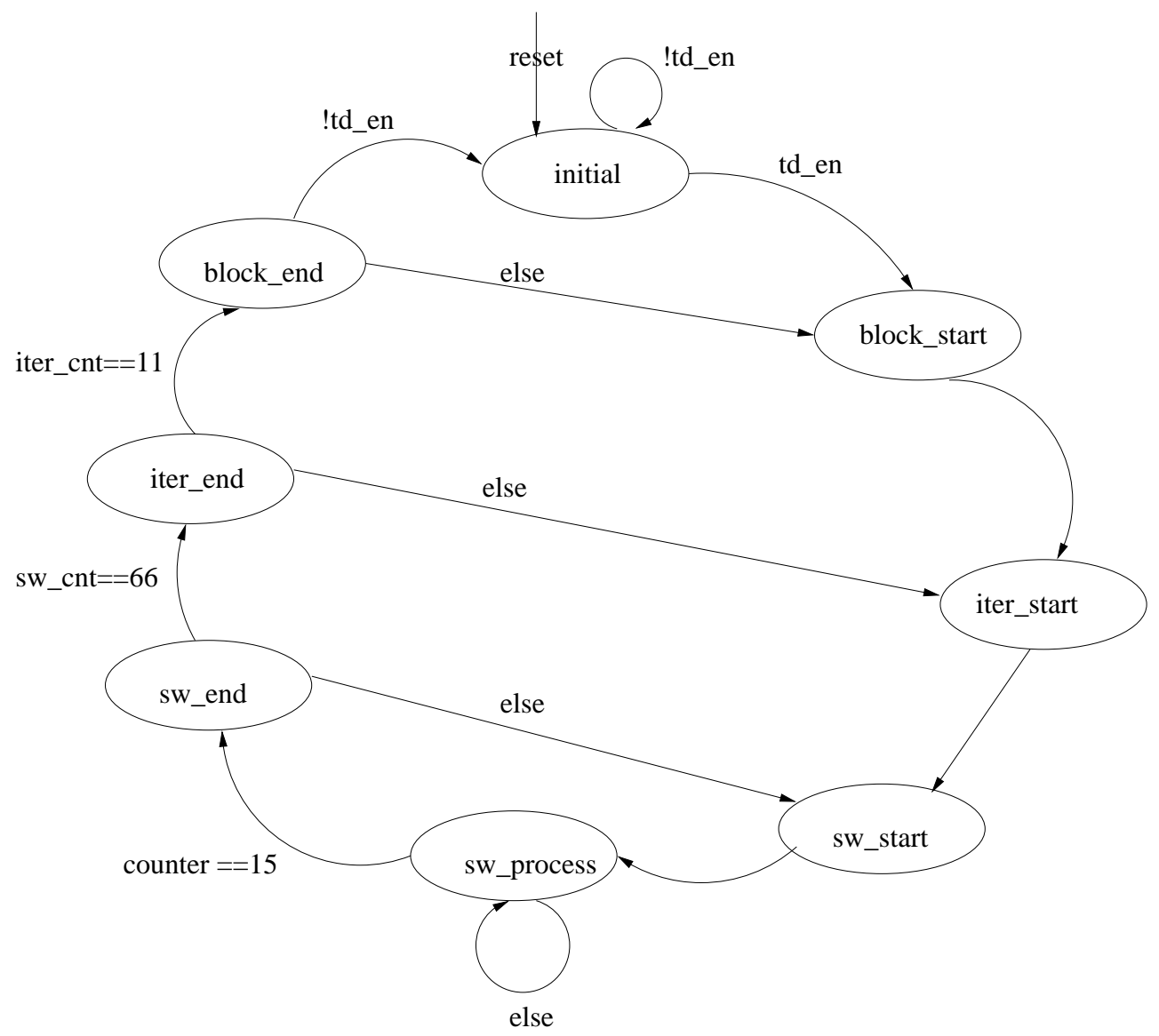

Figure 6.4: Control logic FSM 


\section{Chapter 7}

\section{Optimization Scheme}

This section describes our optimization scheme to reduce the hardware complexity with neglectable degradation to the BER. We optimize the design by select the variables width in the RTL implementation. In most cases, shorter variable width leads to smaller circuit, fast running frequency and lower power consumption. On the other hand, it will also add more quantization errors to the system. As a result, the performance is reduced.

The hardware complexity is measured by the total area of the chip because in digital VLSI design, smaller area design often leads to less power consumption and shorter critical path delay. We use three methods to estimate the hardware complexity:

For performance analysis, we first run an floating-point simulation and get the performance metrics as our reference. As discussed in[14], levelization and scalarization is done to the MAT$\mathrm{LAB}$ code to have a bit-true for every internal variables and a good control to the order during the accumulation. The important signals are grouped into several clusters and assigned to different length of bits. After the fixed-point simulation we will compare the performance metrics with our floating-point simulation results.

\subsection{Theoretical Hardware Complexity Analysis}

For the equalizer, the major hardware consumption is the FIR filters. The first-order estimate of the hardware complexity can be expressed in terms of the multiply-accumulates(MACs) that are 
required[9] to implement the FIR filters. Assume the input bit width for both real and complex part are $n$ bits. We have 4 equalizer modules for 4 transmit antennas and each equalizer contains 4 FIR filters for 4 receive antennas. Each FIR filter have 1 MAC unit and used in a time-multiplexed fashion for 16 taps. The total complexity is $4 \times 4=16$ MACs. It should be noted that the input of the MACs are the concatenation of real and complex part of the input value, i.e., $2 n$ bits. There are 9 memory blocks in the equalizer, one input memory, four coefficient memories and four correlator memories. The input memory contains $2 n \times 4 \times 32$ bits for the sampled data from the RF front-end. Both the coefficient memory and correlator memory have the size of $2 n \times 8 \times 16$ bits. To sum up, the total memory required is $2304 n$ bits.

It is difficult to estimate the sphere decoder in terms of number of adder and multiplier since the sphere searching incorporate a lot of control logic besides arithmetic units. The silicon area of the sphere decoder depends on the bit width for the symbols from the equalizer. We investigate three different bit width for the input symbols: 7 bits, 8 bits and 9 bits, the corresponding bit width for the despread symbol is 14 bits, 16 bits and 18 bits respectively. For each quantization scheme, we run a synthesis for the two major components, the $\mathrm{QR}$ decomposition and the matrix manipulation and get an area report from the EDA tools as shown in Table 7.1. Two sphere decoders are required to process the data from the equalizer and they can share the $\mathrm{QR}$ decomposition module.

Table 7.1: Sphere Decoder Quantization Summary.

\begin{tabular}{|c|c|}
\hline quantization scheme & area $\left(\mu m^{2}\right)$ \\
\hline 7 & 3.19 \\
\hline 8 & 3.42 \\
\hline 9 & 3.87 \\
\hline
\end{tabular}

Assume the bit width for the input of the turbo decoder is $m$, the bit width for the recursion metrics is typical 4 bits larger than the input. The complexity of the turbo decoder is $4 \times 8$ number of $(m+4)$ adders. The memory required is approximately $m \times 1 k \times 6+10 \times 1 k$ bits since the metric memories are much smaller than the input buffers.

\subsection{Critical Signals}

We target two signal sets in our optimization: the sampled input data and the LLR from the sphere decoder. First of all, the input signals from the Radio Frequency front end are very important 
in our implementation since buffers are required to store the input datas and the precision of other signals in the system is greatly depended on these input signals. According to our system level design, the amplitude of input signal is set to 8 , thus 3 bits before the decimal point is required for the input signals. The number of bits after the decimal point affect the system performance and required to be carefully chosen. Let $q . f$ denote the bit width scheme in which totally $q$ bits are used and of which $f$ bits are used after the decimal point for the variables. We simulate the system with three schemes of 7.4, 8.5 and 9.6 and the BER is plotted in Fig. 7.1. We can see the different between 7.4 and 8.5 is as large as $0.4 \mathrm{~dB}$ while the different between 8.5 and 9.6 is much smaller. In addition, the hardware complexity and power consumption of different schemes from the synthesis result of one equalizer module is shown in Table 7.2.

Table 7.2: Input Signal Quantization Summary.

\begin{tabular}{|c|c|c|}
\hline quantization scheme & area $\left(\mu m^{2}\right)$ & power $(\mathrm{mW})$ \\
\hline 7.4 & 0.792 & 21.5 \\
\hline 8.5 & 0.915 & 25.1 \\
\hline 9.6 & 1.125 & 30.0 \\
\hline
\end{tabular}

In addition, the LLR from the Sphere Decoder which contains the soft decoding information of the information bits is critical too. The bit length of the soft LLR will greatly affect the memory required for the buffer and the data path area in the Turbo decoder. We apply investigate three schemes of different bit width of the LLR signal: 12.6, 8.4 and 6.2. As shown in Fig. 7.2, the BER performance between 12.6 and 8.4 is less than $0.1 \mathrm{~dB}$. However, the curve for 6.2 has about 0.3 $\mathrm{dB}$ degradation comparing to 12.6. The hardware complexity and power consumption of different schemes from the synthesis result for the whole chip is shown in Table 7.3.

Table 7.3: LLR Quantization Summary.

\begin{tabular}{|c|c|c|}
\hline quantization scheme & area $\left(\mu m^{2}\right)$ & power(mW) \\
6.2 & 8.12 & 495 \\
\hline 8.4 & 9.49 & 557 \\
\hline 12.6 & 11.22 & 610 \\
\hline
\end{tabular}




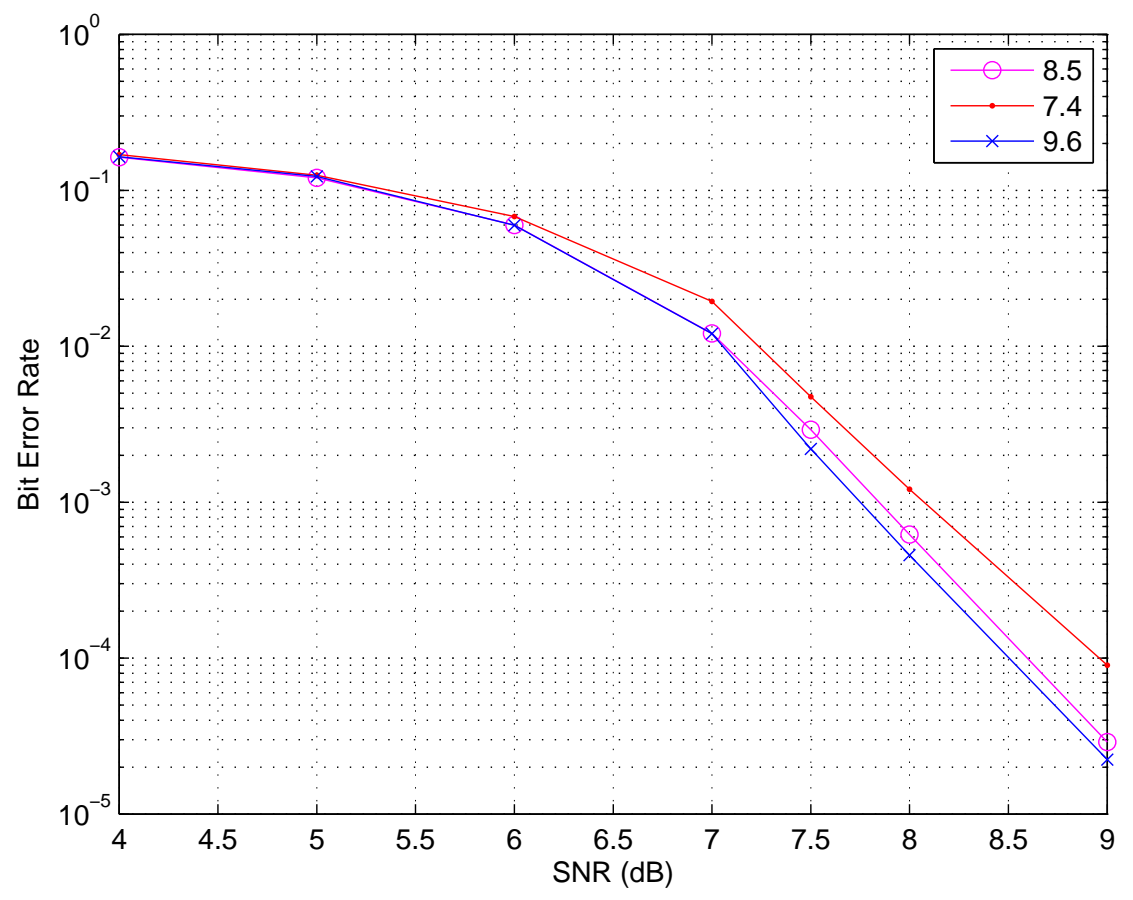

Figure 7.1: Bit error rate of different input schemes.

\section{3 $\quad L_{c}$ Value in Turbo Decoder}

As shown in Fig. 7.2, the BER for scheme 8.4 or 6.2 is even lower than 12.6 at low SNR range. The reason lies in the $L_{c}$ value in the turbo decoder. The channel reliability value $L_{c}$ is derived by $L_{c}=4 * E_{c} / N_{0}$, where $E_{c}$ is the received bit energy and $N_{0}$ is the white Gaussian noise power spectral density. The turbo decoding algorithm requires the channel SNR to calculate $L_{c}$. However,It has been shown[17] that a fixed $L_{c}$ value can be used in a large range of SNR with little performance degradation. We choose the fixed $L_{c}$ value as an exponential of 2 in our design so that we can use shift operation to compute the multiplication of $L_{c}$ and the received information. This value is suitable in the SNR between $6 \mathrm{~dB}$ and $10 \mathrm{~dB}$ according to our simulation. At low SNR, for the scheme of 12.6, since the amplitude of the signal is larger than 8.6 or 6.2, the effective $L_{c}$ value should also be increased. However, the $L_{c}$ value is fixed which leads to a higher BER at low SNR. For wireless application, the desired BER should be less than $10^{-3}$. Thus our target SNR is larger than about $8 \mathrm{~dB}$ in which the fixed $L_{c}$ value can be used. 


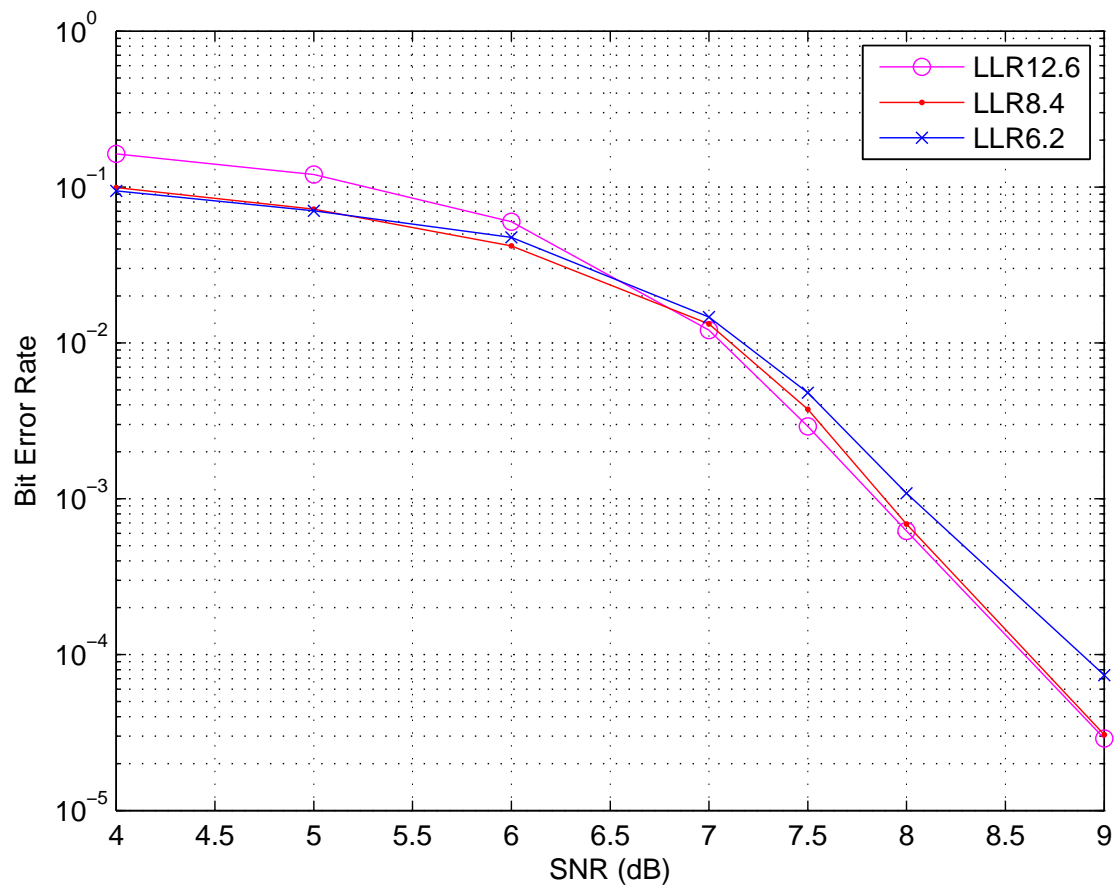

Figure 7.2: Bit error rate of different LLR schemes. 


\section{Chapter 8}

\section{Chip Design Result}

\subsection{Design Flow}

Our MIMO receiver is designed using a hierarchical ASIC design flow. Specifically, a MATLAB implementation of the target MIMO system, including the transmitter and the receiver, is created first. In addition to the hardware verification purpose, the derivation of the MATLAB code provides an efficient way to optimize the MIMO receiver at the algorithm level. Once the MATLAB code is finalized, the MIMO receiver partitioned into several modules and then each module is designed in Verilog hardware description language. The datapath and controller modules are synthesized using design_compiler from Synopsys. The memory modules are generated using the Artisan memory compiler. The layout of our SoC design is created using automatic placement and routing tool soc_encounter from Cadence. The functional verification of the MIMO receiver is performed by simulating the synthesized Verilog code using the output from the MATLAB transmitter model as the stimulus and comparing the results in a bit-by-bit fashion with those of the MATLAB simulation.

\subsection{Chip Performance and Layout Summary}

We have simulated our MIMO receiver to demonstrate its performance. Specifically, the transmitted binary data are generated randomly by the MATLAB model. The transmitted symbols are 
derived based on the MIMO transmitter design described in Section 2.1.1. We model the wireless channel as a frequency selective channel with inter-symbol interference. Addictive white Gaussian noise is introduced into this channel model. We changed the SNR by varying the amplitude of the white Gaussian noise.

Fig. 8.1 shows the BERs of our MIMO receiver using floating point simulation and fixed-point simulation on our receiver. The MIMO receiver is configured with 16QAM modulation.

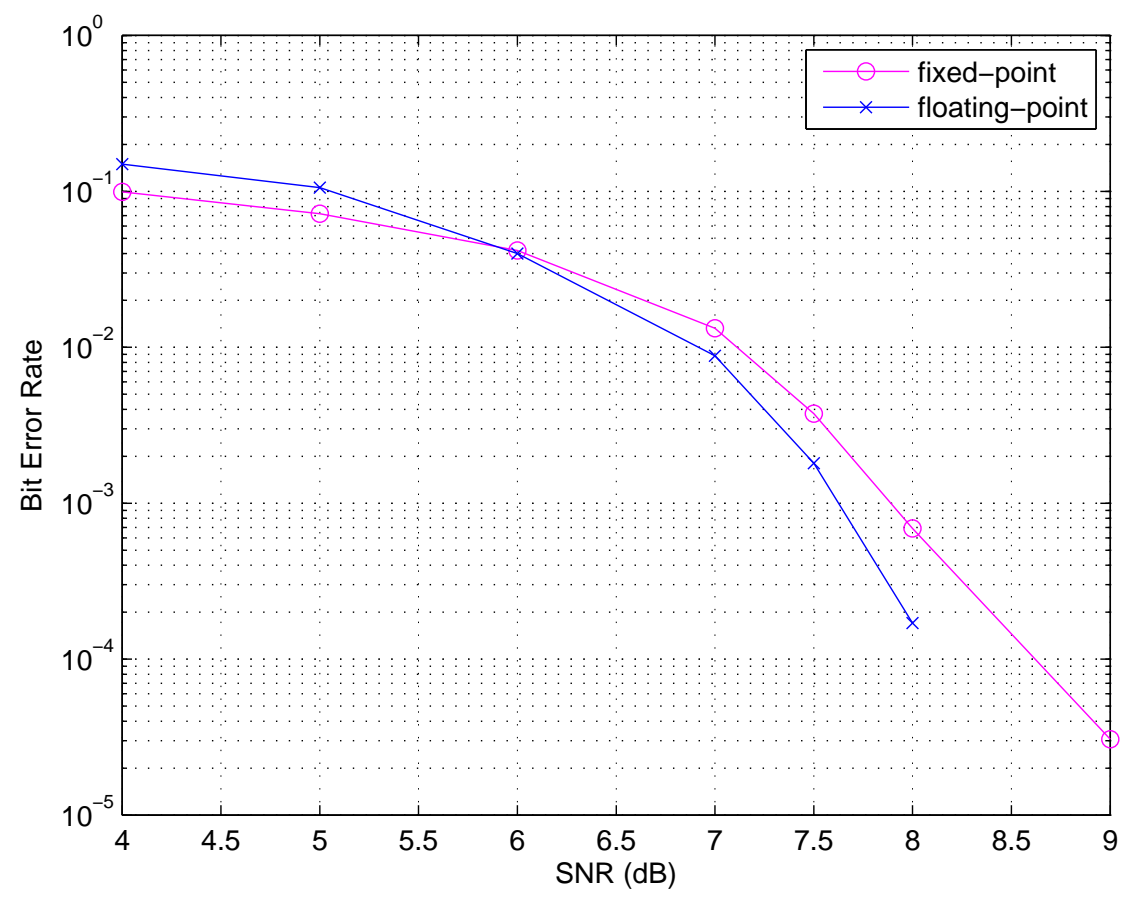

Figure 8.1: Bit error rate of the MIMO receiver.

The layout of the MIMO receiver is shown in Fig. 8.2. As marked in the figure, the left part the core with several lined up SRAM memories is the equalization module. The input buffer is located at the bottom left corner. The sphere decoder module is sit to the right of the equalization module. The channel estimation module is adjacent to both the equalization and sphere decoder module, since this module use the output from equalization and derives the channel matrix for the sphere decoder. The turbo decoder is located at the right side of the core with several memories with various sizes. To sum up, the modules are placed in a fashion that the physical path of the data flow is minimized, which makes the chip easier to meet the tight timing constrain. The SoC chip 


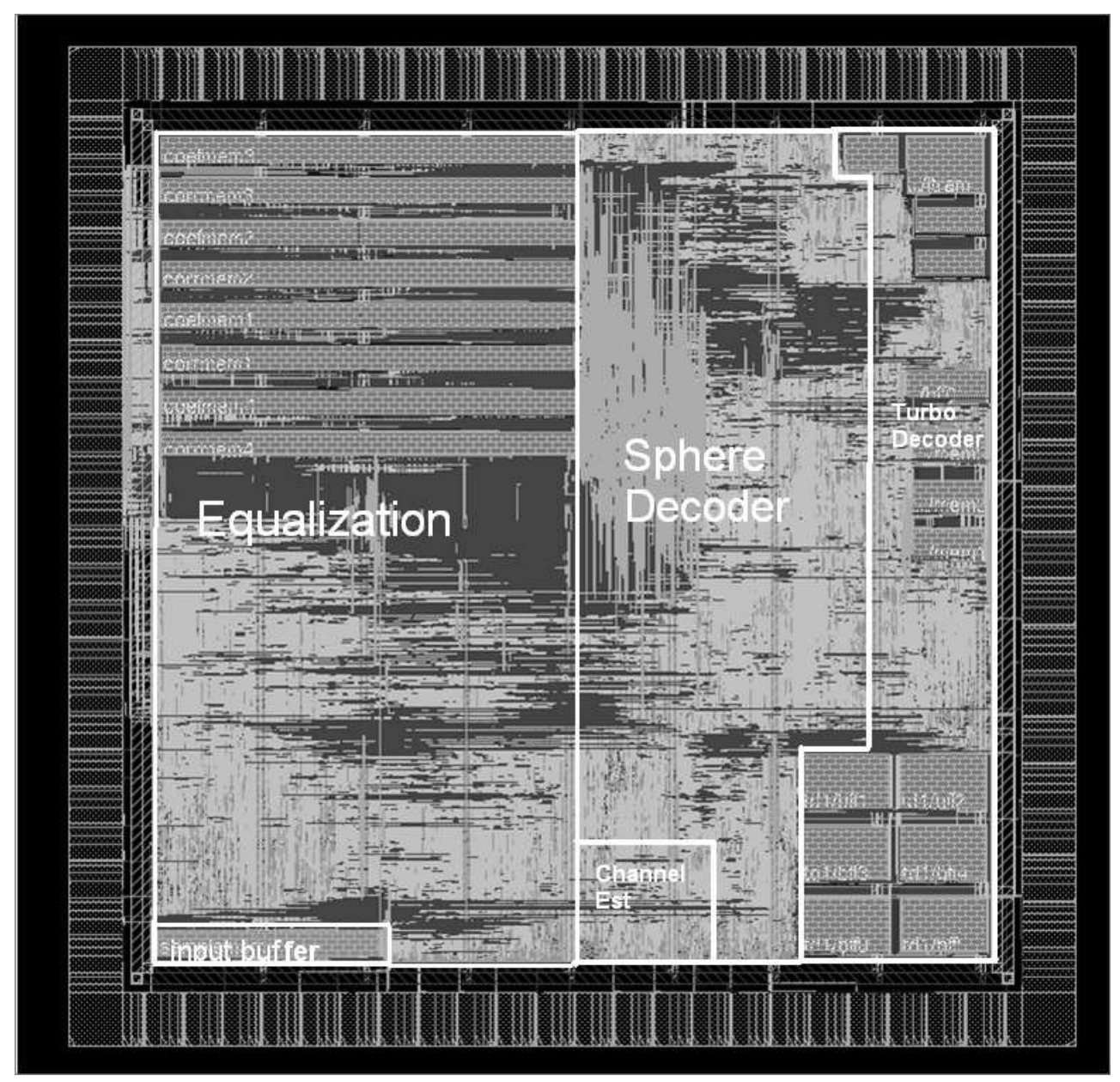

Figure 8.2: Full chip layout.

is designed using a $0.18 \mu \mathrm{m}$ standard cell library with 6 metal layers. It contains 0.92 million logic gates and 23 memory modules. The chip core area is $16.5 \mathrm{~mm}^{2}$. With a power supply of 1.8 volts, the chip can operate at $80 \mathrm{MHz}$ and deliver a throughput of $5 \mathrm{Mbps}$ per user, capable of supporting a $75 \mathrm{Mbps}$ wireless channel with 15 users. The estimated power dissipation is $557 \mathrm{~mW}$. 


\section{Chapter 9}

\section{Conclusions and Future Work}

\subsection{Conclusions}

With the highly increasing demands for high speed and wide bandwidth wireless communication systems, MIMO systems have been considered as one of the key solution for current wireless systems. The hardware design of the MIMO system is more critical for real applications. In this thesis, we present an SoC implementation of a $4 \times 4$ MIMO CDMA receiver that targets at the third generation wireless communication applications. Analysis on the trade-off between the hardware complexity and system performance shows that our implementation can achieve high performance as a BER as low as $5 \times 10^{-3}$ at a SNR of $8 \mathrm{~dB}$. Its low complexity make it feasible for silicon implementation with today's ASIC standard cell technology.

\subsection{Future Work}

We can improve the design in several ways. Firstly, we will verify the design thoroughly using industrial standard tool such as System C or System Verilog. Both function coverage and code coverage should be considered during the verification. Secondly, more modulation schemes other than QPSK and 16QAM should be supported by the MIMO chip. It will be desirable if the chip can change the modulation in real time automatically. Finally, we will reduce the power consumption to extend the battery lifetime for portable device. 


\section{Bibliography}

[1] L. Bahl, J. Cocke, F. Jelinek, and J. Raviv. Optimal decoding of linear codes for minimizing symbol error rate. IEEE Trans. Inform. Theory, IT-20:284-287, March 1974.

[2] S. Benedetto et al. Soft-output decoding algorithms for continuous decoding of parallel concatenated convolutional codes. In IEEE ICC'96, pages 140-152, June 1996.

[3] C. Berrou, A. Glavieux, and P. Thitimajshima. Near shannon limit error-correcting coding and decoding. In ICC'93, pages 1064-1070, May 1993.

[4] M. Bickerstaff et al. A $24 \mathrm{Mb} / \mathrm{s}$ radix-4 LogMAP turbo decoder for 3GPP-HSDPA mobile wireless. In Inter. Solid-State Circuits Conference, March 2003.

[5] A. Burg et al. VLSI implementation of MIMO detection using the sphere decoding algorithm. J. of Solid-States Circuits, 40(7):1566-1577, July 2005.

[6] S. Chen et al. Relaxed k-best MIMO signal detector design and VLSI implementation. IEEE Trans. VLSI Systems, 15(3), March 2007.

[7] L. Davis. Scaled and decoupled cholesky and QR decompositions with application to spherical MIMO detection. In Wireless Communications and Networking, March 2003.

[8] G. J. Foschini. Layered space-time architecture for wireless communication in a fading environment when using multi-element antennas. Bell Lab Tech. J., 1(2):41-59, August 1996.

[9] D. Garrett et al. A $28.8 \mathrm{Mb} / \mathrm{s} 4 \times 4 \mathrm{MIMO} 3 \mathrm{G}$ CDMA receiver for frequency selective channels. J. of Solid-States Circuits, 40(1):320-330, January 2005.

[10] T. Kaiser et al. Protyping for MIMO systems - an overview. In Eusipco 2004, September 2004. 
[11] G. Masera et al. VLSI architectures for turbo codes. IEEE Trans. VLSI Systems, 7(3), September 1999.

[12] P. Murphy et al. A hardware testbed for the implementation and evaluation of MIMO algorithms. In Inter. Conf. on Mobile and Wireless Communication Networks, October 2003.

[13] P. Robertson, E. Villebrun, , and P. Hoeher. A comparison of optimal and sub-optimal map decoding algorithms operating in the log domain. In ICC'95, pages 1009-1013, May 1995.

[14] S. Roy and P Banerjee. An algorithm for trading off quantization error with hardware resources for MATLAB-based FPGA design. IEEE Trans. on Computer, 54(7):886-896, July 2005.

[15] A. van Zelst and T. C. W. Schenk. Implementation of a MIMO OFDM-based wireless LAN system. IEEE Trans. Signal Processing, 52(2), February 2001.

[16] Z. Wang, H. Suzuki, and K. K. Parhi. VLSI implementation issues of turbo decoder design for wireless applications. IEEE SiPS99, pages 503-512, 1999.

[17] Zhongfeng Wang. High performance, low complexity VLSI design of turbo decoders. PhD Thesis, Univeristy of Minnesota, September 2000.

[18] www.airgonetworks.com. 